\title{
Recurrent chylothorax in a woman with primary amenorrhoea
}

\author{
Mangalam Sridhar, Iain Boyle ${ }^{1}$ and Stephen Banham
}

\author{
Department of Respiratory Medicine and ${ }^{1}$ University Department of Medicine (Endocrinology), Glasgow \\ Royal Infirmary, Glasgow, UK
}

\begin{abstract}
Summary: A 47 year old woman receiving oestrogen replacement therapy for primary amenorrhoea presented with recurrent chylothorax. The clinical and radiological features of her illness were characteristic of pulmonary lymphangio-leiomyomatosis, a rare hormone-dependent disease. The condition was treated with pleurodesis and withdrawal of oestrogen therapy.
\end{abstract}

\section{Introduction}

Chylothorax, the collection of chylous fluid in the pleural space is a rare cause of pleural effusion. ${ }^{1}$ The aetiology includes malignancy involving the mediastinal structures causing obstruction of the thoracic duct (especially lymphomas and metastases from gastric carcinoma), surgical and nonsurgical trauma to the thoracic duct, and thrombosis of the jugular and subclavian veins. Other more unusual causes are tuberculosis, filariasis, yellow nail syndrome and lymphangioleiomyomatosis (LAM). ${ }^{2,3}$ The latter condition is a rare cause of chylothorax and lung disease affecting women of child-bearing age. In recent years there has been particular interest in the apparent sexhormone dependence of this condition. ${ }^{4}$ We present here a case of recurrent chylothorax due to LAM, with an unusual clinical course which provides further support for the importance of hormonal manipulation in control of disease progression.

\section{Case report}

A 47 year old school teacher presented with a 3 month history of progressive breathlessness.

Her first contact with the hospital was at the age of 17 when she was investigated for short stature and primary amenorrhoea. Bone age was found to be less than chronological age but thyroid, pituitary function and sex chromosome constitution were normal. The patient was placed on thyroid

Correspondence: M.K. Sridhar, M.B., M.R.C.P.(U.K.)., Department of Respiratory Medicine, Glasgow Royal Infirmary, 16 Alexandra Parade, Glasgow G31 2ES, UK. Accepted: 13 January 1994 hormones as a growth stimulant. Menstruation was induced by oestrogen therapy (piperazine oestrone sulphate $1.5 \mathrm{mg}$ daily). A detailed endocrinological assessment was performed once more at the age of 28 , prior to the patient's proposed emigration to Canada. Cortisol response to insulininduced hypoglycaemia and tetracosactin (Synacthen) were normal. Plasma and urinary gonadotrophin levels were low. There was a subnormal response to a luteinizing hormone releasing test. A diagnosis of hypogonadotrophic hypogonadism was made on the basis of these investigations. A routine chest $\mathrm{X}$-ray performed at this time was reported as showing prominent lung markings at both lung bases but no action was taken as the patient was asymptomatic. The patient remained in good health until the onset of her present complaint, save for intermittent problems associated with oestrogen therapy, which required temporary withdrawal of therapy on a number of occasions. There was no history of thoracic surgery or trauma.

On examination the patient was of short stature (height $135 \mathrm{~cm}$ ) and thin (weight $35 \mathrm{~kg}$ ). There was no finger clubbing or lymphadenopathy. There were signs of a right pleural effusion. A computed tomographic (CT) scan of the thorax confirmed the effusion and showed in addition a small pneumothorax on the right as well as scattered cystic and parenchymal abnormalities in both lower lung fields (Figures 1 and 2). The mediastinum was normal. Aspiration of the pleural effusion revealed a milky white fluid which showed the presence of triglycerides and chylomicrons on biochemical analysis, confirming it to be a chylothorax. Pleural biopsies showed no specific abnormality. Lung function tests performed after plerual aspiration showed poor lung function (forced vital capacity: 1.4 litre; forced expiratory volume in the first 


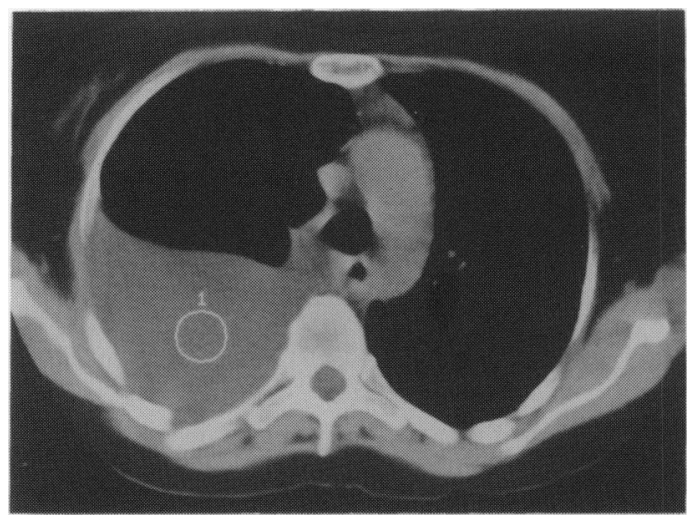

Figure 1 Right-sided chylothorax (computed tomography of thorax).

second: 1.1 litre). A clinical diagnosis of lymphangioleiomyomatosis was made on the basis of the typical clinical features and CT scan appearances. ${ }^{5}$ A lung biopsy was not performed in view of the poor lung function.

Kaolin pleurodesis of the right pleural space was undertaken following failure of conservative management by tube drainage and altered diet. Three weeks later the patient presented with a chylothorax on the left side which was treated in a similar manner. Oestrogen therapy was withdrawn. Lung function tests performed 6 weeks after the second pleurodesis showed a decrease in lung function measured by spirometry (forced vital capacity: 1 litre). At her last review $2 \frac{1}{2}$ years from the initial presentation with a chylothorax, the patient was asymptomatic and in good health. A recent chest $\mathrm{X}$-ray showed prominent lung markings at both lung bases but no evidence of deterioration of the disease. Spirometric values have also remained stable.

\section{Discussion}

Lymphangioleiomyomatosis (LAM) is a disease of women of child-bearing age characterized by proliferation of smooth muscle along a lymphatic distribution, in the absence of cancer. ${ }^{6}$ Although the disease affects mainly the lungs causing spontaneous pneumothorax, chylothorax or rarely haemoptysis, peritoneal involvement is also documented. ${ }^{3}$ In some cases the disease is associated with features of tuberous sclerosis. The disease is rare with fewer than a hundred cases having been reported.

The diagnosis of LAM is usually made on the basis of a lung biopsy but sometimes the distinctive

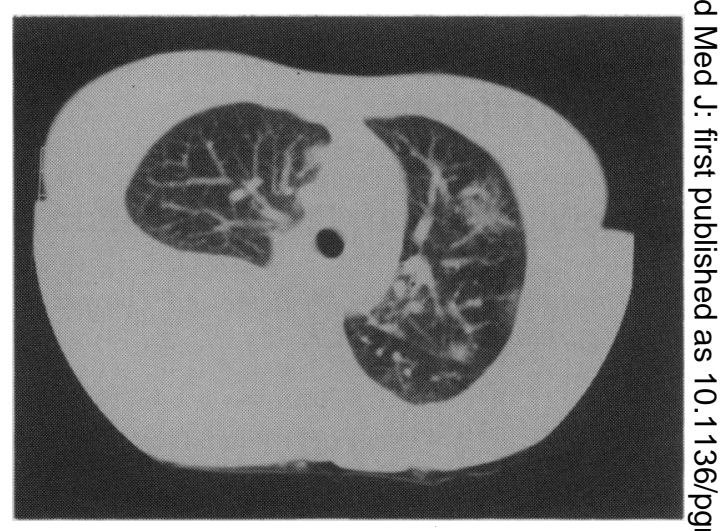

Figure 2 Cystic and parenchymal abnormalities of left lung.

combination of clinical and radiological features, in particular the presence of thin-walled cysts in the $\vec{\sim}$ lung parenchyma on computerized tomography, is $\mathbb{\Phi}$ considered diagnostic. ${ }^{5}$ In our case the presence of $\frac{}{\mathbb{D}}$ a chylothorax, a pneumothorax and pulmonary 3 parenchymal abnormalities in a woman, in the $\underset{\mathbb{D}}{ }$ absence of any other cause for these conditiong, rendered LAM the most likely diagnosis.

Since LAM occurs exclusively in women childbearing age, it is believed to be a hormonet dependent condition. ${ }^{4}$ Oestrogen has been postulated to play a causative role, and treatment has therefore included attempts at hormonal manipulation by oophorectomy, ${ }^{7}$ tamoxifen therapy ${ }^{8}$ and $\stackrel{\odot}{\varnothing}$ progesterone therapy. ${ }^{9}$ The disease is described as $\overrightarrow{\vec{F}}$ being relentlessly progressive with death due to $\frac{\rho}{3}$ respiratory failure occurring within 10 years of diagnosis. ${ }^{10}$

Our case is of interest for a number of reasons. Although oestrogen is strongly implicated in the 3 pathogenesis of LAM and LAM has been des- $\dot{\sigma}$ cribed in post-menopausal women on oestrogen supplementation, ${ }^{3}$ to our knowledge this is the first $\delta$ case of LAM to be reported in a primarily amenorrhoeic woman in whom the only oestrogenic stimu- 의 lus has been entirely exogenous. Also of interest is the relatively long interval of 18 years between the 을. date of appearance of a radiological abnormality $\underset{\sim}{\mathrm{N}}$ and the clinical presentation. We believe that the or slow and atypical progression of the disease was $N$ due to the lack of a sustained oestrogenic stimulus, the patient having been on intermittent oestrogen therapy only. Should the patient continue to remain in a state of clinical remission as she has for $\frac{\varnothing}{\varnothing}$ the past $2 \frac{1}{2}$ years following the withdrawal of $\stackrel{\rho}{+}$ oestrogen therapy, her case would provide direct $D$ evidence for the hormone-dependent nature of LAM. 


\section{References}

1. Macfarlane, J.R. \& Holman, C.W. Chylothorax. Am Rev Respir Dis 1972, 105: 287.

2. Light, R.W. In Pleural Disease. Lea \& Febiger, Philadephia, 1983, pp. 209-210.

3. Taylor, J.R., Ryu, J., Colby, T.V. \& Raffin, T.A. Lymphangioleiomyomatosis: clinical course of 32 patients. $N$ Engl $J$ Med 1990, 323: 1254-1259.

4. Urban, T., Kuttenn, F., Compel, A., Marsac, J. \& Lacromique, J. Pulmonary lymphangiomyomatosis. Follow-up and longterm outcome with antioestrogen therapy. A report of eight cases. Chest 1992, 102: 472-476.

5. Merchant, R.N., Pearson, M.G., Rankin, R.N. \& Morgan, W.K.C. Computerized tomography in the diagnosis of lymphangiomyomatosis. Am Rev Respir Dis 1985, 131: 295-297.
6. Stovin, P.G., Lum, L.C., Flower, C.D., Darke, C.S. \& Beeley, $M$. The lungs in lymphangioleiomyomatosis and in tuberous sclerosis. Thorax 1975, 30: 497-509.

7. Banner, A.S., Carrington, C.B., Emory, W.B. et al. Efficacy of oophorectomy in lymphangiomyomatosis and benign metastasising leiomyoma. N Engl J Med 1981, 305: 204-209.

8. Luna, C.M., Gene, R., Jolly, E.C. et al. Pulmonary lymphangioleiomyomatosis associated with tuberous sclerosis: treatment with tamoxifen and tetracycline pleurodesis. Chest 1985, 88: 473-475.

9. McCarty, K.S. Jr, Mossler, J.A., McLelland, R. \& Sieker, H.O. Pulmonary lymphangiomyomatosis responsive to progesterone. $N$ Engl J Med 1980, 303: 1461-1465.

10. Corrin, B., Leibow, A.A. \& Friedman, P.J. Pulmonary lymphangioleiomyomatosis. Am J Pathol 1975, 79: 348-366. 